\title{
Mechanismen zur
}

\section{Gestaltung erfolgreicher digitaler Plattformen}

Lisa Lohrenz, Simon Michalke, Susanne Robra-Bissantz und Christoph Lattemann

Wirtschaftsinformatik \& Management 2021 • 13 (2): 132-142

https://doi.org/10.1365/s35764-021-00323-0

Angenommen: 21. Januar 2021

Online publiziert: 8. März 2021

(c) Der/die Autor(en) 2021 
Digitale Plattformen werden durch die Digitalisierung immer beliebter. Jedoch fällt auf, dass einige Plattformbetreiber sich am Markt etablieren können, während andere scheitern.

Der Erfolg einer Plattform muss somit bestimmten Kriterien unterliegen, die untersucht werden können. Damit können auch Gestaltungsrichtlinien für das Design von zukünftigen Plattformen vorgegeben werden.

Aufgrund ihrer zunehmenden Marktmacht und Erfolge in verschiedenen Branchen sind digitale Plattformen derzeit ein populäres Thema bei Praktikern und Forschern aus den unterschiedlichsten Bereichen. Digitale Plattformen spielen nicht nur bei dominanten Technologiekonzernen wie Amazon, Apple und Alphabet eine entscheidende Rolle, sondern setzen sich in immer mehr Branchen durch, da sie sich auf den effizienten und effektiven Austausch von Ressourcen zwischen Nutzern fokussieren [1]. Beschleunigt durch die Digitalisierung, fällt jedoch auf, dass einige Plattformbetreiber sich am Markt etablieren können, andere scheitern und wieder andere eine solche Dominanz aufbauen, dass fortwährend geprüft wird, ob sie reguliert werden müssen. Auch für Einzelpersonen wird die Nutzung von digitalen Plattformen von Sorgen um Abhängigkeit, Datenschutz, Betrug und mentaler Gesundheit begleitet. Daher werden aktuell nicht nur Diskussionen um erfolgreiche, sondern auch um Gestaltungsrichtlinien für digitale Plattformen geführt, welche Nutzer bei der Sicherung ihres Wohlbefindens unterstützen [2]. In einer vorangegangenen ausführlichen Literaturstudie [3] konnten wir jedoch in über 1000 Artikeln zu diesem Thema nur eine Handvoll identifizieren, die sich mit der Gestaltung von digitalen Plattformen auseinandersetzen. Die dort dargestellten Gestaltungsrichtlinien beschreiben dabei nur eingeschränkt, wie digitale Plattformen zu designen sind, um Nutzer durch optimal entwickelte Funktionen und Möglichkeiten anzulocken und langfristig zu binden.

\section{Grundlegende Funktionen von digitalen Plattformen}

Die technologischen Fortschritte der letzten Jahrzehnte haben zur Entwicklung von kollaborativen Netzwerken geführt, die stark auf digitale Technologien angewiesen sind [4]. Dies ermöglicht es Organisationen, mit innovativen, informationstechnologischen Strukturen auf Kundenbedürfnisse $\mathrm{zu}$ reagieren. Dadurch entstehen Wettbewerbsvorteile, die zu einer raschen Verbreitung von Plattform-Geschäftsmodellen in vielen Branchen geführt haben [5]. Solche Plattform-Ökosysteme werden „als eine soziotechnische Umgebung von Individuen, Organisationen und digitalen Technologien mit kollaborativen und wettbewerbsorientierten Beziehungen zur gemeinsamen Wertschöpfung über gemeinsame digitale Plattformen" bezeichnet [4, S. 54]. Ausgehend von dieser Definition ist eine digitale Plattform eine zentrale Komponente, die die Co-Creation in Ökosystemen ermöglicht und fördert. $\mathrm{Zu}$ den Nutzern, die an der Co-Creation auf digitalen Plattformen beteiligt sind, gehören Konsumenten, Leistungsanbieter und weitere Unternehmen mit unterschiedlichen Bedürfnissen und Ressourcen. Daher liegt die zentrale Herausforderung nicht unbedingt in der Entwicklung der technologi-
Lisa Lohrenz $z^{1}(\triangle)$

ist wissenschaftliche Mitarbeiterin und Doktorandin am Lehrstuhl für Wirtschaftsinformatik, insbesondere Informationsmanagement an der Technischen Universität Braunschweig. l.lohrenz@tu-braunschweig.de

\section{Simon Michalke ${ }^{2}$}

ist wissenschaftlicher Mitarbeiter und Doktorand am Lehrstuhl für Innovationsmanagement, Wirtschaftsinformatik und International Business an der Jacobs University Bremen. s.michalke@jacobsuniversity.de.

Prof. Dr. Susanne Robra-Bissantz ${ }^{1}$ leitet das Institut für Wirtschaftsinformatik der Technischen Universität Braunschweig und dort den Lehrstuhl Informationsmanagement.

Prof. Dr. Christoph Lattemann ${ }^{2}$ ist Professor für Betriebswirtschaftslehre und Informationsmanagement an der Jacobs University Bremen.

${ }^{1}$ Technische Universität Braunschweig, Institut für Wirtschaftsinformatik, insbs. Informationsmanagement

Braunschweig, Deutschland

${ }^{2}$ Jacobs Universität Bremen, Bremen, Deutschland 


\section{Zusammenfassung}

- Digitale Plattformen gewinnen zunehmend an Einfluss und Dominanz in verschiedenen Branchen.

- Die Kombination von Erkenntnissen aus der Psychologie und der Dienstleistungsforschung liefert vielversprechende Ansätze für die Gestaltung und Weiterentwicklung von Plattformen im B2B- und B2C-Bereich.

- Insgesamt wurden Experten zu 24 Plattformen aus verschiedenen Sektoren befragt und in die Gestaltung eingebunden. schen Architektur, sondern darin, heterogenen Gruppen eine nachhaltige Umgebung für ihre Aktivitäten zu bieten.

Da auf digitalen Plattformen in der Regel zwei oder mehr Nutzergruppen agieren, beispielsweise Lieferanten, Käufer, Plattformanbieter oder Drittdienstleister, besteht eine weitere Herausforderung darin, eine kritische Masse an Nutzern für die Plattform zu gewinnen, welche zudem die verschiedenen Gruppen ausbalancieren [6]. Zu diesem Zweck müssen digitale Plattformen einen kontinuierlichen Zustrom neuer Nutzer durch Senkung der Barrieren und/oder durch die Einbindung von externen Nutzern und Communitys unterstützen. Wenn Nutzer keine fortlaufenden Verbesserungen und neue Wachstumsmöglichkeiten auf etablierten digitalen Plattformen wahrnehmen, profitieren neue, konkurrierende Anbieter dadurch, dass sie erfolgreiche und fortschrittliche Funktionen relativ günstig imitieren können und so eine attraktive Alternative zu etablierten Plattformen darstellen. Da Wechselkosten und Eintrittsbarrieren von digitalen Plattformen bewusst niedrig gehalten werden, um neue Nutzer zu gewinnen, sind die möglichen Gegenmaßnahmen für etablierte Plattformen, abseits der fortlaufenden Bindung durch Verbesserungen, begrenzt.

\section{Entwicklung von Gestaltungsrichtlinien für digitale Plattformen}

Forschungen in der Psychologie haben gezeigt, dass sowohl die Motivation als auch das Wohlbefinden von Nutzern von der Befriedigung bestimmter psychologischer Bedürfnisse abhängig sind. Diese wirken sich ebenfalls auf das nachhaltige Engagement von Nutzern aus. Auch wenn diese Verknüpfung bekannt ist, werden die psychologischen Grundbedürfnisse, die nachweislich diese Ergebnisse vermitteln, nur selten berücksichtigt [2]. Darüber hinaus gibt es Plattformmechanismen, die dazu verleiten, dass sich Nutzer länger als notwendig auf der digitalen Plattform aufhalten und somit nicht auf das Wohlergehen abzielen [7].

Somit stellt sich die Frage:

Wie kann Technologie und hier insbesondere digitale Plattformen gestaltet werden, um Wohlbefinden zu unterstützen?

Um Wohlbefinden zu fördern, können die drei Konstrukte Autonomie, Kompetenz und Zugehörigkeit betrachtet werden. Diese stammen aus der Selbstbestimmungstheorie, welche von den Psychologen Deci und Ryan eingeführt wurde [8]. Sie schlugen vor, dass die menschliche Motivation auf einer Vielzahl verschiedener emotionaler Bedürfnisse und interner/externer Einflüsse beruht. Im Wesentlichen konzentriert sich die Theorie darauf, wie sehr die Handlungen, Entscheidungen und das Verhalten eines Individuums durch Selbstmotivation und Entschlossenheit bestimmt werden.

Wir wollen damit nicht aussagen, dass sich menschliche Handlungen auf lediglich drei Konstrukte zusammenfassen lassen. Jedoch sind Autonomie, Kompetenz und Zugehörigkeit die am gründlichsten erforschten und als wesentlich und vorhersagend für das Wohlbefinden identifiziert und belegt worden [8]. 


\section{Autonomie}

Bei der Autonomie geht es um das Bedürfnis nach freiem Willen. Die Plattformnutzer müssen das Gefühl haben, dass sie Entscheidungen treffen und Handlungen ausführen, die mit ihren Überzeugungen und Meinungen übereinstimmen. Das bedeutet nicht unbedingt, dass sie unabhängig sein und an Online-Trainingskursen zum Selbststudium teilnehmen müssen, sondern dass sie das Gefühl haben sollten, sich bei der Benutzung der Plattform selbst treu zu bleiben und freie Entscheidungen treffen können.

\section{Zugehörigkeit/soziale Eingebundenheit}

Plattformnutzer müssen das Gefühl haben, dass sie dazugehören und Teil einer größeren Online-Community sind. Es geht darum, mit Gleichgesinnten verbunden zu sein und zu wissen, dass man mit ihnen auf eine sinnvolle Weise interagieren kann. Dazu gehört auch, sich um andere zu kümmern und das Gefühl zu haben, dass Meinungen und Gedanken respektiert werden.

\section{Kompetenz}

Die Plattformnutzer müssen Vertrauen in ihre eigenen Fähigkeiten haben sowie in der Lage sein, eine Aufgabe zu erledigen oder bestimmte Fähigkeiten zu erlernen. Sie sollten ein Gefühl von Kompetenz und Vertrauen haben, dass sie das Ergebnis einer Situation kontrollieren können. Kurz gesagt, sie müssen genau wissen, was passieren wird, wenn sie eine bestimmte Handlung ausführen oder eine Entscheidung treffen. Kompetenz kommt nicht nur von den Informationen und Fähigkeiten, die sie lernen, sondern auch von persönlichen Erfahrungen.

\section{Gestaltungsrichtlinien für digitale Plattformen}

Um das Problem mit der erfolgreichen Gestaltung von digitalen Plattformen unter der Prämisse der gleichzeitigen Steigerung des Wohlbefindens der Nutzer zu lösen, stützen wir unser Forschungsprojekt auf das DesignScience-Research-Paradigma [9]. Das übergreifende Ziel dabei ist die Generierung von sogenanntem Designwissen, welches sich auf die Entwicklung von Artefakten mit einer Verbesserung für den Nutzer konzentriert.

Hierfür haben wir Experteninterviews mit 24 Gründer*innen, Manager*innen und CEOs erfolgreicher Plattformen im B2B- und B2CBereich aus der DACH-Region durchgeführt (Tab. 1). Die Interviewfragen wurden dabei unter Berücksichtigung der Erkenntnisse der vorangegangenen Literaturanalyse [3] entwickelt.

Als Ergebnis definieren wir vier Gestaltungsrichtlinien, die sich aus den durchgeführten Interviews ableiten [10, 11]. Diese vier Richtlinien verfolgen unterschiedliche Ziele und lassen sich durch 13 identifizierte Mechanismen unterstützen. Die Mechanismen berücksichtigen die verschiedenen Konstrukte Autonomie, Zugehörigkeit und Kompetenz und sorgen so dafür, dass die Nutzung der Plattform selbstbestimmt bleibt, welches das Wohlergehen des Nutzers in den Vordergrund stellt. Dabei sind die Mechanismen nicht ausschließlich nur einem Ziel oder nur einem Konstrukt zugeordnet, son- 
dern können mehrmals vorkommen. Die vier Ziele (I-IV) mit ihren Mechanismen (1-13) und zugeordneten Konstrukten sind zusammengefasst in Abb. 1.

\section{I: Nutzer anziehen und an sich binden}

Da bestimmte Angebote erst durch eine hinreichend große Anzahl an Nutzern realisiert werden können, benötigt eine digitale Plattform eine kritische Masse an Nutzern, um für andere attraktiv zu sein. Das erste Ziel muss daher sein, Nutzer für die digitale Plattform zu gewinnen und zu binden.

Die Maßnahme die Sichtbarkeit erhöhen (1) umfasst daher Aktivitäten wie Marketingkampagnen durchführen, um die Bekanntheit zu steigern oder auch Beteiligung auf Social-Media-Kanälen, welches die Zugehörigkeit fördert.

\section{Tab. 1 Interviewte Plattformexpert*innen}

\begin{tabular}{|c|c|c|c|}
\hline Plattform & Fokus & & Position \\
\hline Agrirouter & Landwirtschaft & $\mathrm{B} 2 \mathrm{~B}$ & Produktmanager*in \\
\hline Animus & Nachbarschaft & $\mathrm{B} 2 \mathrm{~B} / \mathrm{C}$ & Unternehmensentwicklungsmanager*in \\
\hline Care & Kinderbetreuung & $\mathrm{B} 2 \mathrm{~B} / \mathrm{C}$ & Unternehmensentwicklungsmanager*in \\
\hline Compacer & Prozessautomatisierung & $\mathrm{B} 2 \mathrm{~B}$ & Abteilungsleiter*in \\
\hline Craftnote & Handwerksdienstleistungen & $\mathrm{B} 2 \mathrm{~B}$ & CEO \\
\hline DearEmployee & Betriebliches Gesundheitsmanagement & $\mathrm{B} 2 \mathrm{~B}$ & CEO \\
\hline DS Screening & Betriebliches Gesundheitsmanagement & $\mathrm{B} 2 \mathrm{~B}$ & CEO \\
\hline Einkaufshelden & Einkauf im Einzelhandel & $\mathrm{B} 2 \mathrm{~B} / \mathrm{C}$ & Gründer*in \\
\hline ExtraSauber & Reinigungsleistungen & $\mathrm{B} 2 \mathrm{~B} / \mathrm{C}$ & Gründer*in \\
\hline Homesitter-Eu & Hausbetreuung & $\mathrm{B} 2 \mathrm{C}$ & Gründer*in \\
\hline Itonics & Innovationsplattform & $\mathrm{B} 2 \mathrm{~B}$ & Unternehmensentwicklungsmanager*in \\
\hline Jobruf & Crowd Sourcing & $\mathrm{C} 2 \mathrm{C}$ & Gründer*in \\
\hline Klickrent & Maschinenverleih & $\mathrm{B} 2 \mathrm{~B}$ & Unternehmensentwicklungsmanager*in \\
\hline Mercateo & Handelsplattform & $\mathrm{B} 2 \mathrm{~B}$ & Segmentmanager*in \\
\hline MyFeelix & Finanzen und Versicherung & $\mathrm{B} 2 \mathrm{C}$ & Vertriebsmanager*in \\
\hline MyHammer & Handwerksdienstleistungen & $\mathrm{B} 2 \mathrm{~B} / \mathrm{C}$ & CEO \\
\hline MyHelpBuddy & Sprachhelfer & $\mathrm{B} 2 \mathrm{~B} / \mathrm{C}$ & Gründer*in \\
\hline Nebenan & Nachbarschaftshilfe & $\mathrm{B} 2 \mathrm{C}$ & Gründer*in \\
\hline Notfallmamas & Kinderbetreuung & $\mathrm{B} 2 \mathrm{~B} / \mathrm{C}$ & Gründer*in \\
\hline Pengueen & Kooperationsplattform & $\mathrm{B} 2 \mathrm{~B} / \mathrm{C}$ & CEO \\
\hline Sandkasten & Studenteninitiativen & $\mathrm{B} 2 \mathrm{C}$ & Gründer*in \\
\hline Sqetch & Textilbranche & $\mathrm{B} 2 \mathrm{~B}$ & CEO \\
\hline Wucato & Beschaffungsplattform & $\mathrm{B} 2 \mathrm{~B}$ & CEO \\
\hline Yoopies & Kinderbetreuung & $\mathrm{B} 2 \mathrm{~B} / \mathrm{C}$ & Vertriebsmanager*in \\
\hline
\end{tabular}


Sind Nutzer bereit, der Plattform beizutreten, kann durch die Unterstützung des Onboardings (2) die Kompetenz gefördert und die Austrittswahrscheinlichkeit gesenkt werden. Ein Experte sagt „Wir haben kleine Videos und Tutorials zu Registrierungsprozessen oder Funktionalitäten" und so können die Nutzer mit der Plattform vertraut gemacht werden.

Neben der richtigen Hilfestellung für neue Nutzer kommt es auch darauf an, dass sie möglichst schnell Vertrauen in die Plattform aufbauen. Der gezielte Einsatz von vertrauensbildenden Symbolen und Aktivitäten (3) kann dabei helfen, ein Gefühl von Sicherheit bei der Nutzung zu vermitteln. Wie ein Experte betont, hilft es auch, „ehrlich und transparent zu sein. Wir sind nah und zugänglich." Als beispielhafte Aktivitäten können hier die menschliche Moderation von Inhalten genannt werden, welches das Zugehörigkeitsgefühl stützt, oder beispielsweise TÜV-Siegel, welche durch die transparente Darstellung dem Nutzer Autonomie signalisieren. Hierbei kann auch die Umsetzung fairer Kosten- und Ertragsmodelle (4) zum einen die Autonomie und zum anderen das Zugehörigkeitsgefühl durch Mitentscheidung der Nutzer fördern und den Einstieg in die Plattform attraktiver machen. Ein Experte erklärte zum Beispiel: „Wir verlangen keine Gebühr, wenn man ein Inserat schaltet oder Profil erstellt, bis zu dem Punkt, ..., ist die Nutzung

\begin{tabular}{|c|c|c|}
\hline \multicolumn{3}{|c|}{ Abb. 1 Gestaltungsziele, Mechanismen und Konstrukte } \\
\hline & Mechanismus & Konstrukt \\
\hline & (1) Sichtbarkeit erhöhen & Zugehörigkeit \\
\hline \multirow{2}{*}{$\begin{array}{l}\text { I: Plattformnutzende } \\
\text { anziehen und an sich binden }\end{array}$} & (2) Unterstützung des Onboardings von neuen Nutzenden & Kompetenz \\
\hline & $\begin{array}{l}\text { Einsetzen von vertrauensbildenden Symbolen und } \\
\text { Aktivitäten }\end{array}$ & $\begin{array}{l}\text { Autonomie, } \\
\text { Zugehörigkeit }\end{array}$ \\
\hline \multirow{3}{*}{$\begin{array}{l}\text { II: Gemeinsames Wachstum } \\
\text { erzielen }\end{array}$} & (4) Umsetzung fairer Kosten- und Ertragsmodelle & $\begin{array}{l}\text { Autonomie, } \\
\text { Zugehörigkeit }\end{array}$ \\
\hline & Teilnahme an persönlichen Treffen & Zugehörigkeit \\
\hline & $\begin{array}{l}\text { (6) Verbindung zwischen bestehenden und neuen Partnern } \\
\text { herstellen }\end{array}$ & Zugehörigkeit \\
\hline \multirow{3}{*}{$\begin{array}{l}\text { III: Interaktion und Co- } \\
\text { Creation fördern }\end{array}$} & 7) Nutzende als gleichberechtigte Partner behandeln & $\begin{array}{l}\text { Autonomie, } \\
\text { Zugehörigkeit }\end{array}$ \\
\hline & Offen sein für neue Lösungen & Autonomie \\
\hline & (9) Auf Benutzerfreundlichkeit achten & Kompetenz \\
\hline \multirow{4}{*}{$\begin{array}{l}\text { IV: Wettbewerbsfähigkeit } \\
\text { durch Serviceinnovationen } \\
\text { steigern }\end{array}$} & Neue, nützliche Funktionen entwickeln & $\begin{array}{l}\text { Kompetenz, } \\
\text { Zugehörigkeit }\end{array}$ \\
\hline & (11) Funktionen für den Austausch von Ressourcen definieren & Kompetenz \\
\hline & (12) Feedback-Kanäle für Innovationen nutzen & $\begin{array}{l}\text { Autonomie, } \\
\text { Zugehörigkeit }\end{array}$ \\
\hline & $\begin{array}{l}\text { (13) Nutzende vom langfristigen Bestehen der Plattform } \\
\text { überzeugen }\end{array}$ & Autonomie \\
\hline
\end{tabular}


komplett kostenlos." Eine offene und transparente Preisgestaltung signalisiert den Nutzern somit, dass eine faire Behandlung im Ökosystem der Plattform einen hohen Stellenwert hat. Persönliche Treffen (5) stellen eine gute Möglichkeit dar, bekannter zu werden, die Nutzer persönlich kennenzulernen und die soziale Eingebundenheit zwischen den Plattformnutzern herzustellen. Zusätzlich ist der Aufbau einer Verbindung zwischen bestehenden und neuen Partnern (6) von Vorteil, da der Bekanntheitsgrad und die vermeintliche Vertrauenswürdigkeit etablierter Partner genutzt werden können und so die soziale Eingebundenheit weiter gefördert wird. So gab ein Experte an, dass man „... eher auf etablierte Unternehmen setzt, die bereits Vertrauen aufgebaut haben." Und dass es eine große Herausforderung sei, „... eine Firma zu gründen, die noch keinen Namen hat.“

\section{II: Gemeinsames Wachstum erzielen}

Um die allgemeine Wettbewerbsposition einer digitalen Plattform in sich ständig verändernden Märkten zu festigen, müssen Probleme und Bedürfnisse aller Nutzer adressiert werden, damit ein gemeinsames Wachstum erreicht werden kann.

Um auf zukünftige Herausforderungen, zum Beispiel gesetzliche Änderungen, vorbereitet zu sein, sollten die bereits vorgestellten Maßnahmen persönliche Treffen (5) und die Verbindung von bestehenden und neuen Partnern (6) genutzt werden.

Zusätzlich kann die Umsetzung fairer Kosten- und Ertragsmodelle (4) ein Wachstum kleinerer Partner überhaupt erst möglich machen. Als weitere Maßnahme konnten wir herausarbeiten, dass Nutzer als gleichberechtigte Partner behandelt (7) werden müssen, was impliziert, dass die Bedürfnisse aller Beteiligten einer Plattform berücksichtigt und Probleme offen angesprochen werden. Somit können das Zugehörigkeitsgefühl, aber auch die wahrgenommene Eigenkompetenz und somit Autonomie gesteigert werden. „Ein respektvoller Umgang auf Augenhöhe sorgt für ein gutes Verhältnis.“ Fühlen sich die Nutzer respektiert, sind sie zudem offener für eine Zusammenarbeit und eine Weiterempfehlung der Plattform. Bei der Zusammenarbeit mit den Nutzern können neue Ideen entstehen und Lösungen für Probleme gefunden werden. Daher sollten Aktivitäten eingesetzt werden, die darauf abzielen, offen für neue Lösungen zu sein (8). Durch die freiwillige Beteiligung an der Zusammenarbeit, entwickelt sich das Gefühl von Autonomie und langfristige Vorteile, wie z. B. geringere Innovationskosten und die Gewissheit, dass die Plattform sich an Nutzerbedürfnisse anpasst, entstehen.

\section{III: Interaktion und Co-Creation fördern}

Die Co-Creation von Werten wird nur durch die Integration von Ressourcen durch verschiedene Nutzer ermöglicht. Daher sind die Einbeziehung und das Engagement aller Nutzer wesentliche Treiber für den Erfolg einer digitalen Plattform.

Durch die Einbindung bestehender und neuer Partner (7) in Gestaltungsprozesse können die Plattformen besser auf die individuellen Bedürfnisse 
abgestimmt werden. Um die bestmögliche Zusammenarbeit zu erreichen, sollte die Plattform so gestaltet sein, dass eine intuitive Bedienbarkeit gewährleistet ist (9). Daher sollte sich das Design an bereits bekannten Plattformen orientieren, um die Vertrautheit mit diesen Plattformen zu nutzen und dem Nutzer das Gefühl von Kompetenz zu geben. Dazu gehört auch die bereits beschriebene Gestaltung eines einfachen Prozesses zur Unterstützung des Onboardings (2). Das Wissen um die Möglichkeiten und Grenzen der Plattform hilft dabei, Bereiche für die gemeinsame Erstellung (Co-Creation) neuer und nützlicher Funktionen zu identifizieren (10). Dabei kann durch Innovationsworkshops Kompetenz und soziale Eingebundenheit gefördert werden. Wie ein Experte beschreibt: „... Innovationen sind eigentlich immer neue Funktionen für uns. Sie können auch von uns kommen, da wir immer bestrebt sind, unsere Plattform weiterzuentwickeln. "Durch klar definierte Regeln, welche Handlungskonsequenzen vorhersehbar machen und damit die Kompetenz der Nutzer fördern, müssen dann die geeigneten Möglichkeiten zum Ressourcenaustausch (11) geschaffen werden, sowohl virtuell als auch in der realen Welt, damit die Plattform einen Mehrwert bietet.

\section{IV: Verbesserung der Wettbewerbsfähigkeit durch Innovation}

Da sich die Märkte ständig verändern und die Anforderungen der Nutzer steigen, müssen digitale Plattformen nachhaltige Innovationen sicherstellen.

Dies kann durch die bereits vorgestellten Maßnahmen, das Anbieten von neuen, nützlichen Funktionen (10) und das Offensein für neue Lösungen (8) erreicht werden.

Zusätzlich können Feedback-Kanäle für Innovationen (12), zum Beispiel Kundenhotlines, Click-Stream-Daten und UX-Tests genutzt werde. Die Experten berichten, dass Innovationen vorwiegend von den Nutzern getrieben werden: „Das können Kunden sein, die uns eine kurze E-Mail mit Verbesserungsvorschlägen schicken. “ Durch den persönlichen Austausch wird das Zugehörigkeitsgefühl und durch die Freiwilligkeit die Autonomie gefördert.

Die Einbindung bestehender und neuer Partner (6) ermöglicht es den Plattformbetreibern Kompetenzen einzubringen, die sonst im eigenen Unternehmen entwickelt werden müssten, und so können sie schneller innovieren. Dies verschafft ihnen einen Vorteil gegenüber potenziellen Wettbewerbern und signalisiert den Nutzern, dass die Plattformen zukunftssicher sind (13), also schnell auf Marktveränderungen oder gesetzliche Anforderungen reagieren können, und stärkt damit die Autonomie.

Die Befragung mit den Plattformexperten aus dem B2B- und B2C-Kontext hat gezeigt, dass beide Sektoren auf ähnliche Maßnahmen und insbesondere auf die gleichen grundlegenden Mechanismen setzen, um Nutzer für ihre Plattformen zu gewinnen und zu halten. Bei einer stichprobenartigen Befragung von zehn Experten bestätigten diese, trotz unterschiedlicher sektoraler Zugehörigkeit, die Anwendbarkeit, Relevanz und Nützlichkeit der vorgestellten Gestaltungsrichtlinien. Die Unterschiede zwischen B2Cund B2B-Plattformen liegen hauptsächlich in der eher formalen und durch

\section{Handlungsempfehlungen}

Um nachhaltig erfolgreiche Plattformen zu gestalten, sollten vier Ziele verfolgt werden:

1. Nutzer anziehen und an sich binden

2. Gemeinsames Wachstum erzielen

3. Interaktion und Co-Creation fördern

4. Verbesserung der Wettbewerbsfähigkeit durch Innovationen 


\section{Kernthesen}

- Jede mehrseitige Plattform sollte Nutzerzahlen, gemeinsame Wachstumsmöglichkeiten, Interaktion und Innovationen gezielt durch dedizierte Mechanismen fördern.

- Alle Mechanismen fördern ein oder mehrere Aspekte von Autonomie, Zugehörigkeit oder Kompetenz.

- Nutzerwohlbefinden und somit hohe Bindung an Plattformen wird durch die Förderung von wahrgenommener Autonomie, Zugehörigkeit und Kompetenz erhöht.
Verträge geprägten Sicherstellung von Autonomie und fairen Bedingungen für die Nutzer. Bei den Plattformen im Bereich B2C haben wir beispielsweise festgestellt, dass die Experten mehr in Maßnahmen investieren, die das Zugehörigkeitsgefühl und einen guten Umgang der Nutzer untereinander fördern. Dies ist nicht verwunderlich, da hierunter auch beispielsweise Plattformen im Bereich Nachbarschaftshilfe zu finden sind, die zu einem erheblichen Teil daraus bestehen, dass die Nutzer miteinander kommunizieren. Im Gegensatz dazu haben B2B-Plattformen kaum Interesse, dass eine Kommunikation außerhalb der eigentlichen Transaktionen stattfindet, die oftmals bereits hochgradig standardisiert und vertraglich genau definiert sind. Weiter setzen B2B-Plattformen mehr Maßnahmen ein, die zu einem Kompetenzerwerb der Nutzer führen. Da B2B-Plattformen oft komplexer sind und Einarbeitung brauchen, sind geeignete Hilfestellungen für Nutzer hier von entscheidender Bedeutung.

\section{Implikationen für die Praxis}

Wir wollen kein Online-Tool sein, dass die Leute ... online zieht, sondern eine Plattform, die ein nützliches Online-Tool ist und immer auch offline einen echten Mehrwert bringt (Geschäftsführung einer der befragten Plattformen).

Basierend auf 24 Experteninterviews, haben wir vier Gestaltungsprinzipien entwickelt, die grundlegende Ziele und Mechanismen für die Entwicklung von digitalen Plattformen und deren Funktionen beschreiben. Bisher wurden solche Ergebnisse selten mit Erkenntnissen bezüglich der Auswirkungen auf das Wohlbefinden der Nutzer verglichen. Um dem nachzukommen, haben wir die vier Gestaltungsrichtlinien daraufhin untersucht, welchen Beitrag sie für die wahrgenommene Autonomie, Kompetenz und Zugehörigkeit leisten können. Diese Erkenntnisse entstammen der Befragung von Experten von am Markt erfolgreichen digitalen Plattformen aus diversen Sektoren in der DACH-Region. Unsere Ergebnisse bieten eine Informationsgrundlage für strategische und operative Entscheidungen, sowohl für etablierte Plattformbetreiber als auch für solche, die sich in den frühen Phasen der Planung oder bereits in der Implementierung befinden.

Interessierte finden auf der Projektwebseite Informationen zu bisherigen Ergebnissen (www.bedien.org), welche sich insbesondere mit der Digitalisierung personennaher Dienstleistungen beschäftigen [12].

Danksagung. Dieser Beitrag ist Teil des vom Bundesministerium für Bildung und Forschung geförderten Verbundprojekts „BeDien - Begleitforschung Personennahe Dienstleistungen“ (Förderkennzeichen: 02K17A080$81)$.

Funding. Open Access funding enabled and organized by Projekt DEAL. 
Open Access Dieser Artikel wird unter der Creative Commons Namensnennung 4.0 International Lizenz veröffentlicht, welche die Nutzung, Vervielfältigung, Bearbeitung, Verbreitung und Wiedergabe in jeglichem Medium und Format erlaubt, sofern Sie den/die ursprünglichen Autor(en) und die Quelle ordnungsgemäß nennen, einen Link zur Creative Commons Lizenz beifügen und angeben, ob Änderungen vorgenommen wurden.

Die in diesem Artikel enthaltenen Bilder und sonstiges Drittmaterial unterliegen ebenfalls der genannten Creative Commons Lizenz, sofern sich aus der Abbildungslegende nichts anderes ergibt. Sofern das betreffende Material nicht unter der genannten Creative Commons Lizenz steht und die betreffende Handlung nicht nach gesetzlichen Vorschriften erlaubt ist, ist für die oben aufgeführten Weiterverwendungen des Materials die Einwilligung des jeweiligen Rechteinhabers einzuholen.

Weitere Details zur Lizenz entnehmen Sie bitte der Lizenzinformation auf http://creativecommons.org/licenses/by/4.0/deed.de.

\section{Literatur}

[1] Lusch, R. F., \& Nambisan, S. (2015). Service innovation: a service-dominant logic perspective. MIS Quarterly, 39(1), 155-175.

[2] Peters, D., Calvo, R. A., \& Ryan, R. M. (2018). Designing for motivation, engagement and wellbeing in digital experience. Frontiers in Psychology. https:// doi.org/10.3389/fpsyg.2018.00797.

[3] Fischer, S., Lohrenz, L., Lattemann, C., \& Robra-Bissantz, S. (2020). Critical design factors for digital service platforms-A literature review. Research papers, Bd. 85.

[4] Senyo, P. K., Liu, K., \& Effah, J. (2019). Digital business ecosystem: literature review and a framework for future research. International Journal of Information Management, 47, 52-64.

[5] Hein, A., Böhm, M., \& Krcmar, H. (2019). Digitale Plattformen. In M. H. Dahm \& S. Thode (Hrsg.), Strategie und Transformation im digitalen Zeitalter (S. 181-199). Wiesbaden: Springer.

[6] Gawer, A., \& Cusumano, M. A. (2014). Industry platforms and ecosystem innovation: platforms and innovation. Journal of Product Innovation Management, $31,417-433$.

[7] Havi, N. S., \& Samaha, M. (2017). The relations among social media addiction, self-esteem, and life satisfaction in university students. Social Science Computer Review, 35(5), 576-586.

[8] Ryan, R. M., \& Deci, E. L. (2017). Self-determination theory: basic psychological needs in motivation, development, and wellness. New York: Guilford.

[9] Hevner, A. R., March, S. T., Park, J., \& Ram, S. (2004). Design science in information systems research. MIS Quarterly, 28, 75-105.

[10] Lohrenz, L., Michalke, S., Robra-Bissantz, S., \& Lattemann, C. (2021). Fostering visibility, commitment and trust on digital platforms: insights into personal engagement platforms from the DACH region. In Proceedings of the 54th Hawaii International Conference on System Sciences (HICSS).

[11] Michalke, S., Lohrenz, L., Lattemann, C., \& Robra-Bissantz, S. (2021). Design knowledge for digital business ecosystems: towards design principles for digital service platforms. In Baumann (Hrsg.), Handbook on digital business ecosystems. 
[12] Robra-Bissantz, S., Lattemann, C., Guerrero, R., Lux, A. M., Redlich, B., \& Fischer, S. (2020). Der Mensch als Teil der Innovation - Eine „Service Canvas“ als anwendungsorientierter Bezugsrahmen. In Bruhn \& Hadwich (Hrsg.), Automatisierung und Personalisierung von Dienstleistungen. Wiesbaden: Springer Gabler. 